\title{
一种基于非对称逆布局模型的彩色图像表示方法 ${ }^{*}$
}

郑运平 ${ }^{+}$, 陈传波

(华中科技大学 计算机科学与技术学院,湖北 武汉 430074)

\section{A Color Image Representation Method Based on Non-Symmetry and Anti-Packing Model}

\author{
ZHENG Yun-Ping ${ }^{+}, \quad$ CHEN Chuan-Bo \\ (College of Computer Science and Technology, Huazhong University of Science and Technology, Wuhan 430074, China) \\ + Corresponding author: Phn: +86-27-87792260, Fax: +86-27-87792251, E-mail: zypdragon@163.com, http://www.hust.edu.cn
}

Zheng YP, Chen CB. A color image representation method based on non-symmetry and anti-packing model. Journal of Software, 2007,18(11):2932-2941. http://www.jos.org.cn/1000-9825/18/2932.htm

\begin{abstract}
With the concept of the packing problem, this paper presents a color image representation method based on the non-symmetry and anti-packing pattern representation model (NAM). By describing the NAM and the method of the binary-bit plane decomposition (BPD) for the color image, a novel NAM-based representation algorithm for the color image is proposed. Also, the total data amount of the algorithm is analyzed. The theoretical and experimental results presented in this paper show that the NAM-based representation method can reduce the data storage much more effectively than the hierarchical structural linear quadtree-based representation method and is a better method to represent the color image pattern. The method is a new research area with respect to the color pattern representation and is valuable for the theoretical research and potential practical values such as decreasing the storage room, increasing the transmission speed, quickening the process procedure, matching pattern, and so on.

Key words: image representation; color image; NAM (non-symmetry and anti-packing pattern representation model); BPD (binary-bit plane decomposition); linear quadtree; anti-packing problem; image complexity; hierarchical data structure
\end{abstract}

摘 要: 借助于 Packing 问题的思想, 提出了一种基于非对称逆布局的模式表示模型(non-symmetry and antipacking pattern representation model,简称 NAM)的彩色图像表示方法.通过描述 NAM和彩色图像的二进制位平面分 解(binary-bit plane decomposition,简称 BPD)方法,给出了一种全新的基于 NAM 的彩色图像表示算法,并对算法的总 数据量进行了分析.理论分析和实验结果均表明,与流行的基于分层结构的线性四元树的彩色图像表示方法相比,基 于 NAM 的表示方法能够更有效地减少数据存储空间,是彩色图像模式表示的一种良好方法.这种方法可以应用于 彩色图像模式表示的各个方面,在降低存储空间、提高传输速度、加快处理过程、模式匹配等方面具有良好的理论 参考意义和实际应用价值.

关键词: 图像表示;彩色图像;NAM(non-symmetry and anti-packing pattern representation model);二进制位平面分 解;线性四元树;逆布局问题;图像复杂度;分层数据结构

\footnotetext{
* Supported by the National High-Tech Research and Development Plan of China under Grant No.2006AA04Z211 (国家高技术研究发 展计划(863))

Received 2006-10-14; Accepted 2007-01-24
} 
分层数据结构在计算机视觉、机器人、计算机图形学、图像处理、模式识别等领域里是非常重要的区域 表示方法 ${ }^{[1-4]}$.传统的、直观的区域表示方法是二维数组表示,但由于图像处理在各方面的广泛应用和其他学科 的飞速发展,对图像处理的研究提出了处理速度和存储空间上的更高要求. 这样,二维数组的空间效率及其“样 点-样点”的运算方式已不能适应发展的需要,取而代之的应该是既紧凑又便于做各种图像处理运算的表示方 法 $^{[5-7]}$. 图像的分层表示是分层表示的一种重要形式 ${ }^{[8]}$,这种表示形式不仅在空间上更具紧凑性,而且许多图像 处理运算都可以在分层表示的基础上快速实现 ${ }^{[9]}$.分层数据结构之所以有效,可以归结于它的层次结构和“块块”运算方式. 在时间上, 用这种表示形式做图像处理运算所要求的工作量正比于图像中被分割的具有相同灰级 的块数, 而不是图像中的样点数, 并且其运算过程更加简单, 只归结于对树结构的追迹, 这样就提高了执行时间 的效率. 在空间上, 它利用了图像数据的二维相关性, 因而极大地消除了图像数据的冗余度. 四元树是图像分层 表示的一种形式,它是基于图像数组的四元分割. 四元树表示是研究得最早, 也是研究得最多的一种分层表示形 式 ${ }^{[10-12]}$. 早期的四元树表示都是基于指针的四元树结构,为了进一步减少存储空间,Gargantini ${ }^{[13]}$ 消除了指针方 案,提出了称为线性四元树的表示方法.一般情况下,线性四元树可节省 $66 \%$ 的存储空间;特殊情况下,可节省高 于 $90 \%$ 的存储空间. 线性四元树的建立, 在四元树表示的历史中是一个很大的转折. 从现状来看, 图像分层表示主 要集中在降低图像处理运算的复杂性和向更宽的范围扩展上, 理论上的成果很多, 运用于实际的也不少, 并越来 越多 ${ }^{[14-18]}$.

然而,尽管上述的分层数据结构表示有许多优点,但是它们过于强调分割的对称性,因此不是最优的表示方 法.借助于 Packing 问题的思想, 以寻找分割最大化的非对称分割方法为目标,本文提出了一种基于非对称逆布局 的模式表示模型(non-symmetry and anti-packing pattern representation model,简称 NAM)的彩色图像表示方法.该 方法与流行的基于线性四元树的彩色图像表示方法相比,不仅前者的子模式数远小于后者的节点数,而且前者的总 数据量也远小于后者的总数据量, 因此, 前者能够更有效地减少数据存储空间. 本文的表示方法在数据表示和操作 上均优于线性四元树的表示方法,是彩色图像模式表示的一种好方法.

\section{1 非对称逆布局的模式表示模型(NAM)描述}

非对称逆布局的模式表示模型是 Packing 问题的一个反问题.结合诸如图像、语音、文本、视频等处理的 需要, 本文给出一种新的模式表示方法.NAM 的基本思想是,给定一个已经布局好了的容器(模式)和 $n$ 个预先定 义的不同形状的物体( $n$ 个子模式),现在要从这个布局好了的容器(给定的模式)中抽出这些物体(子模式), 用这 些物体(子模式)的组合来表示已布局的容器(给定的模式).

本文的非对称(non-symmetry)概念是指布局或者逆布局(匹配)的格局不是对称的,是相对于分层结构而提 出来的, 这是由于本文的方法主要是与对称形的分层结构进行比较研究的.对于图像模式而言,Freeman 编码是 非对称的,树形分层编码(如二元树、四元树、八元树、以及 $N$ 元树等)都是对称的.

下面是 NAM 的抽象描述.

设原模式为 $\Gamma$, 恢复后的非失真模式为 $\Gamma^{\prime}$, 失真模式为 $\Gamma^{\prime \prime}$, 则 $\mathrm{NAM}$ 是由 $\Gamma$ 到 $\Gamma^{\prime}$ 的一个非失真变换,或者是由 $\Gamma$ 到 $\Gamma^{\prime \prime}$ 的一个失真变换:

其中, $T()$ 是正向变换函数,或称为编码函数.

$$
\Gamma^{\prime}=T(\Gamma), \Gamma^{\prime \prime} \approx T(\Gamma)
$$

正向编码过程为

$$
\Gamma^{\prime}=\bigcup_{j=1}^{n} p_{j}\left(v, A \mid A=\left\{a_{1}, a_{2}, \ldots, a_{m_{i}}\right\}\right)+\varepsilon(d) .
$$

其中, $\Gamma^{\prime}$ 是编码后合成的模式; $P=\left\{p_{1}, p_{2}, \ldots, p_{n}\right\}$ 是预先定义的子模式集合, $n$ 是子模式的类型数, $p_{j} \in P$ 是 $P$ 中第 $j$ 个 子模式; $v$ 是 $p_{j}$ 的值, $A$ 是 $p_{j}(1 \leq j \leq n)$ 子模式的参数集合, 或者是通过智能分析选取的子模式集合; $a_{i}\left(1 \leq i \leq m_{i}\right)$ 是子模式 
$p_{j}$ 的形状参数集合, $m$ 是 $p_{j}$ 的编号; $\varepsilon(d)$ 是残渣模式(容器中的垃圾), $d$ 为残渣空间尺度的阈值.

失真模式 NAM 为

显然有

$$
\Gamma^{\prime \prime}=\bigcup_{j=1}^{n} p_{j}\left(v, A \mid A=\left\{a_{1}, a_{2}, \ldots, a_{m_{i}}\right\}\right)
$$

本文重点讨论基于 NAM 的彩色图像模式的表示方法.

\section{2 彩色图像的二进制位平面分解(binary-bit plane decomposition, 简称 BPD)方法}

彩色图像的二进制位平面分解方法简称为彩色图像的 BPD 方法.本节我们来讨论如何将一幅彩色图像分 解为多个二值图像的方法, 即如何将一幅彩色图像和多个二值图像对应起来. 在具体介绍彩色图像的 BPD 方法 之前,我们先给出一些相关的定义.

\section{1 相关定义}

定义 1 (二值图像模式的复杂度 $C F$ ). $C F=N_{L Q T B} / N_{f b}$, 其中, $N_{L Q T B}$ 为此二值图像用线性四元树表示时的黑色节 点数, $N_{f b}$ 为此图像的像素总数.显然有 $0<C F \leq 1$.

定义 2 (灰度图像模式的复杂度 $C \boldsymbol{p}$ ). $C p=N_{L Q T} / N_{f}$, 其中, $N_{L Q T}$ 为此灰度图像用线性四元树表示时的总块数, $N_{f}$ 为此图像的像素总数.显然有 $0<C p \leq 1$.

定义 3(彩色图像模式的复杂度 $C c) . C c=\left(N_{r}+N_{g}+N_{b}\right) /\left(3 N_{f}\right)$, 其中, $N_{r}, N_{g}$ 和 $N_{b}$ 分别为彩色图像分解后的 3 幅代 表 $r, g, b$ 颜色分量的灰度图像用线性四元树表示时的总块数; $N_{f}$ 为单幅灰度图像的像素总数.显然有 $0<C c \leq 1$.

定义 4(位平面二值图像). 该图像是一幅多灰度值(二进制表示)的某一位面所组成的一幅二值图像,即由该 位面复制而来的二值图像, 用 $B P_{i}$ 来表示, 其中, $i=0,1, \ldots, m-1 . B P_{i}(x, y)$ 是对应地址为 $(x, y)$ 的颜色,即 “ 0 ”和 “ 1 ”, 或 “white”和“black”. 虽然这里的“ 0 ”和“ 1 ”与一般二值图像的“ 0 ”和“ 1 ” 有不同的实际意义,但这里我们还是等同 看待.

\section{2 彩色图像的BPD方法}

彩色图像的 BPD 方法的基本思想是:首先将彩色图像分解为 3 幅代表 $r, g, b$ 颜色分量的灰度图像, 然后对每 一幅分量图像进行灰度图像的 BPD. 灰度图像的 BPD 具体方法及其某些性质通过下面的几个定理给出.

定理 1. 给定任意具有 $K=2^{m}(m>1)$ 个灰级的灰度图像 $M P$, 存在 $m$ 个二值图像 $B P_{0}, B P_{1}, \ldots, B P_{m-1}$, 满足下面的 关系:

$$
M P=\sum_{i=0}^{m-1} 2^{i} B P_{i}
$$

其中, $B P_{i}$ 是从图像 $M P$ 中灰级值的第 $i$ 个二进制位平面复制而得到的二值图像.并规定:如果第 $i$ 位为 $0($ 或 1$)$, 则 $B P_{i}$ 对应的那个像素值也为 0 (或 1).

证明:给定 $M P$ 中的一个像素 $M P(x, y)$,其灰级值为 $g$,且 $g$ 的二进制表示为

$$
g=\left(b_{m-1} b_{m-2} \ldots b_{0}\right)_{2}
$$

即有

$$
g=2^{m-1} b_{m-1}+2^{m-2} b_{m-2}+\ldots+2^{0} b_{0}=\sum_{i=0}^{m-1} 2^{i} b_{i}=M P(x, y)
$$

另一方面, 由定理中的规定可知, $b_{i}$ 正好是 $B P_{i}(x, y)$ 的值. 这样, 将上式中的 $M P(x, y)$ 换成 $M P$, 将 $b_{i}$ 换成 $B P_{i}(x, y)$, 然后换成 $B P_{i}$, 这就是式(1).

知道了 $M P$ 和 $B P_{i}$ 之间的关系后, 剩下的问题就是如何进行分解. 实际上没有必要去对位面一一进行分解, 直接 对 $M P$ 进行操作就可以分解. 为此, 有下面的定理 2.

定理 2. 给定一个二进制位面 $i$ 和 $M P$ 中的一个像素 $M P(x, y)$, 它的灰级值为 $g$, 如果满足条件 


$$
2^{i} \leq g \bmod 2^{i+1}<2^{i+1}, 0 \leq i<m
$$

则 $B P_{i}(x, y)=1$; 否则, $B P_{i}(x, y)=0$.

证明:设 $g=\left(b_{m-1} b_{m-2} \ldots b_{0}\right)_{2}$, 考虑 3 种情况:

第 1 种情况: $2^{i} \leq g<2^{i+1}$.

在这种情况下, $g \bmod 2^{i+1}=g$,式(4)满足.此时, $b_{m-1}, \ldots, b_{i+1}$ 是 $0, b_{i}$ 是 $1, b_{i-1}, \ldots, b_{0}$ 是 0 或 1 . 这说明(由定理 1 ) $B P_{i}(x, y)=1$;

第 2 种情况: $0 \leq g<2^{i}$.

在这种情况下, $g \bmod 2^{i+1}=g$,式(4)条件不满足. 此时, $b_{m-1}, \ldots, b_{i+1}$ 是 $0, b_{i}$ 是 $0, b_{i-1}, \ldots, b_{0}$ 是 0 或 1 . 这说明 $B P_{i}(x, y)=0$;

第 3 种情况: $2^{i+1} \leq g<2^{m}$.

在这种情况下, $g \bmod 2^{i+1}=\left(0 \ldots 0 b_{i} \ldots b_{0}\right)_{2}$, 若 $b_{i}=1$, 即为第 1 种情况, 即式 $(4)$ 满足, $B P_{i}(x, y)=1$; 若 $b_{i}=0$, 即为第 2 种 情况,即式(4)不满足, $B P_{i}(x, y)=0$.

由于在 3 种情况下定理 2 都成立,因此说明定理 2 成立.

关于灰度图像的 BPD,有一个下面正要利用的重要性质.我们用如下的定理来陈述这一性质.

定理 3. $B P_{i}$ 的复杂性 $C F\left(B P_{i}\right)$ 小于 $M P$ 的复杂性 $C p(M P)$. 换句话说,对 $B P_{i}$ 的逆布局所得到的子模式数少 于对 $M P$ 的四元分割所得到的块数, 即 $\sum_{i=0}^{m-1} N_{r}(i)<N_{L Q T}$, 其中, $N_{r}(i)$ 表示逆布局后第 $i$ 个位面的子模式总数, $N_{L Q T}$ 为图像 $M P$ 用线性四元树表示时的总块数.亦即 $B P_{i}$ 扩展或保持了 $M P$ 的块状结构.

证明:给定 $M P$ 和 $B P_{i}$ 中的两个像素, 比如 $M P\left(x_{1}, y_{1}\right), M P\left(x_{2}, y_{2}\right)$ 和 $B P_{i}\left(x_{1}, y_{1}\right), B P_{i}\left(x_{2}, y_{2}\right)$. 如果 $M P\left(x_{1}, y_{1}\right)$ 和 $M P\left(x_{2}, y_{2}\right)$ 有相同的灰级值,则由定理 1 可知, $B P_{i}\left(x_{1}, y_{1}\right)$ 和 $B P_{i}\left(x_{2}, y_{2}\right)$ 有相同的灰级值. 如果 $M P\left(x_{1}, y_{1}\right)$ 和 $M P\left(x_{2}, y_{2}\right)$ 的 灰级值不同,则由定理 1 可知, $B P_{i}\left(x_{1}, y_{1}\right)$ 和 $B P_{i}\left(x_{2}, y_{2}\right)$ 可能有相同的颜色,也可能有不同的颜色.例如, $M P\left(x_{1}, y_{1}\right)=5$ 和 $M P\left(x_{2}, y_{2}\right)=4$, 则有 $B P_{0}\left(x_{1}, y_{1}\right)=1$ 和 $B P_{0}\left(x_{2}, y_{2}\right)=0$. 又如, $M P\left(x_{1}, y_{1}\right)=5$ 和 $M P\left(x_{2}, y_{2}\right)=7$, 则有 $B P_{0}\left(x_{1}, y_{1}\right)=1$ 和 $B P_{0}\left(x_{2}, y_{2}\right)=1$. 这就说明了 $B P_{i}$ 能够扩展或保持 $M P$ 的块状结构,即 $\sum_{i=0}^{m-1} N_{r}(i)<N_{L Q T}$, 从而证明了定理 3 的成立.

综上所述, 定理 1 指出了一幅灰度图像(灰度级参数为 $m$ )与 $m$ 幅位平面二值图像之间的关系. 定理 2 给出了 分解一幅灰度图像的 BPD 方法.定理 3 保证了这种分解方法的空间有效性.

\section{3 彩色图像的BPD方法的实例及其分析}

如图 1 所示,彩色图像在Peppers的大小为 $2^{9} \times 2^{9} \times 3$, 灰度级参数 $m$ 为 8. 由图 1(a)可知,彩色图像的复杂度 $C c$ 为 0.985 , 而 3 幅代表 $r, g, b$ 颜色分量组成的灰度图像的复杂度 $C p$ 分别为 $0.9925,0.9824$ 和 0.981 9,分别如 图 1(b)、图 1(c)和图 1(d)所示.这表明彩色图像的复杂度与其对应的灰度图像的复杂度是大致相同的.但是,3 个色 彩分量中的任何一幅灰度图像,不妨以图 1(c)为例, 在其进一步分解为 8 幅位平面二值图像后(如图 2 所示), 这 8 幅图像的复杂度 $C F$ 均小于原始的灰度图像的复杂度 $C p=0.9824$, 并且面位号越高, 其复杂性越低. 实验也证明了 理论的正确性.

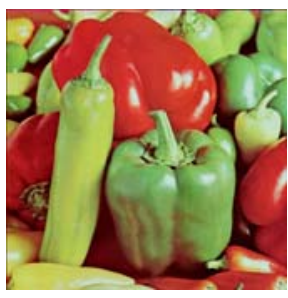

(a) $C c=0.9856$

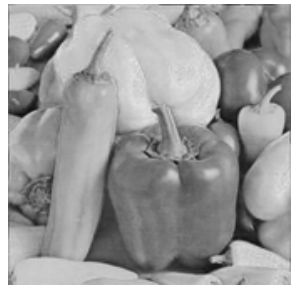

(b) $C p=0.9925$

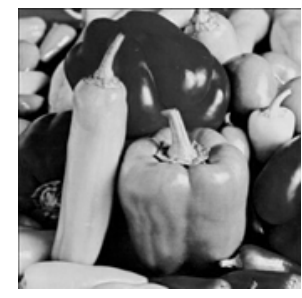

(c) $C p=0.9824$

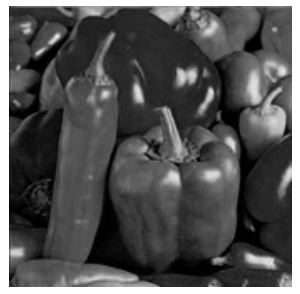

(d) $C p=0.9819$

Fig.1 A color image Peppers and its three gray images by three-color components $r, g, b$ respectively 图 1 彩色图像 Peppers 和它的 $r, g, b$ 颜色分量组成的灰度图像 


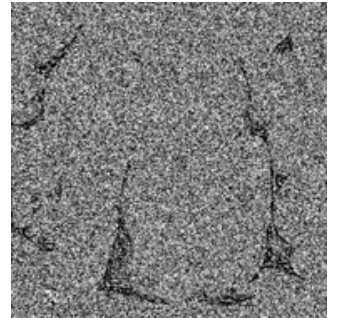

(a) $B P_{0}(C F=0.4530)$

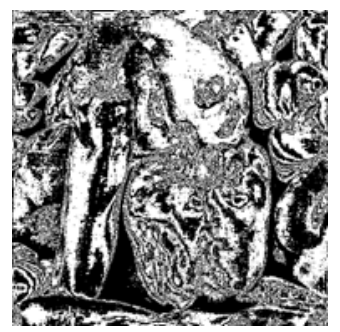

(e) $\mathrm{BP}_{4}(C F=0.2590)$

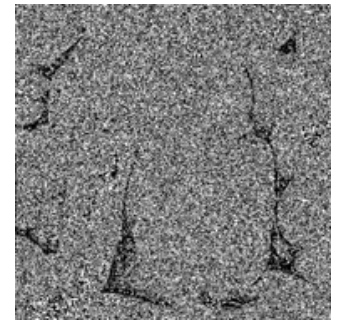

(b) $B P_{1}(C F=0.4478)$

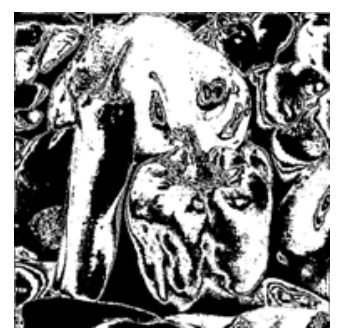

(f) $\mathrm{BP}_{5}(C F=0.1617)$

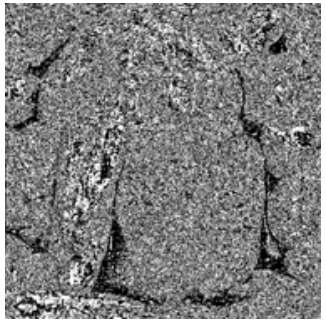

(c) $B P_{2}(C F=0.4194)$

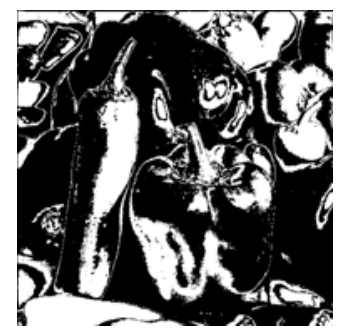

(g) $B P_{6}(C F=0.1038)$

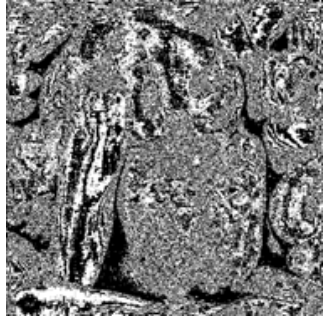

(d) $B P_{3}(C F=0.352$ 2)

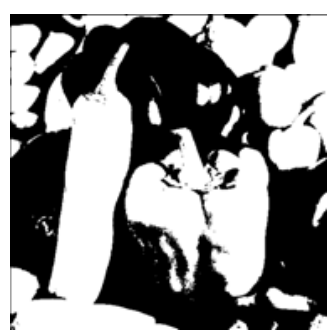

(h) $B P_{7}(C F=0.0381)$

Fig.2 Eight binary-bit plane images after decomposing Fig.1(c) by BPD method

图 2 图 1(c)经过 BPD 方法分解后的 8 幅位平面二值图像

从图 2 不难看出,位面号越低,图像的纹理越复杂;而位面号越高,图像的块状性越好.这是由于低位反映的 是图像信息的细小变化,高位反映的是图像的能量信息.无论是哪幅位平面二值图像,其块状性至少保持或者优 于图 1(c)的块状性.

\section{3 基于 NAM 的彩色图像表示的编解码算法}

基于 NAM 的彩色图像表示的编解码算法中均用到了 $K$ 码变换规则,因此在描述算法之前,有必要对 $K$ 码 变换规则进行介绍.

\section{$3.1 K$ 码变换规则}

设已经布局好了的图像模式用 $F=\{f(x, y)\}$ 来表示, 为简单起见, 假定 $F=\{f(x, y)\}$ 为二值图像, 即 $f(x, y) \in\{0,1\}$, 并 且 $F=\{f(x, y)\}$ 的大小为 $2^{n} \times 2^{n}$.

令 $x=\left(x_{n-1} x_{n-2} \ldots x_{1} x_{0}\right)_{2}, y=\left(y_{n-1} y_{n-2} \ldots y_{1} y_{0}\right)_{2}$, 构造一个一维的坐标变量 $k$, 有

$$
k=\left(y_{n-1} x_{n-1} y_{n-2} x_{n-2} \ldots y_{1} x_{1} y_{0} x_{0}\right)_{2} \text {. }
$$

这样就将一个二维图像模式转换为一个保持块状性质的一维序列,即 $F=\{f(x, y)\}=\{g(k)\}$, 简称这种一维的表示 为 $K$ 码.这样做的好处在于子模式的块状性质会更好,可以大量减少子模式的数量.

由二维到一维的降维变换称为 $K$ 码正变换,记为 $k=K(x, y)$. 由一维到二维的升维变换称为 $K$ 码的反变换,记为 $(x, y)=K^{-1}(k) . K$ 码的正变换和反变换都是二进制数按位拼接过程. $K$ 码反映了图像的块状特征, 这种块状特征有利于 提高图像表示的效率.

\section{2 基于NAM的彩色图像模式的逆布局算法}

本算法中被逆布局的子模式对象是任意大小的矩形 $p=\{$ rectangle $\mid$ rectangle $=L \times W\}$.已经布局好的彩色图像模 式为 $C I P$, 大小为 $2^{n} \times 2^{n} \times 3$, 其分解后的 3 幅灰度图像模式为 $M P[1], M P[2]$ 和 $M P$ [3], 大小均为 $2^{n} \times 2^{n}$, 灰度级别参数为 $m$, 任何一幅灰度图像的 $m$ 幅位平面二值图像为 $B P_{i}$, 其中, $0 \leq i \leq m-1$. 为方便起见,假定“ 0 ”为“black”,即黑色,“ 1 ”为 “white”,即白色.黑色表示区域,白色表示背景点.本算法只记录“black”像素点.由定理 3 可知,经二进制位平面分解 后的二值图像模式具有更好的块状性质,可以大量减少子模式的数量.因此,矩形的匹配算法遵循 $K$ 码方式. 由于这 种算法是可逆的,因而给出了相应的解码算法. 
下面将分别给出编解码算法的具体步骤.

编码算法的具体步骤:

$/ *$ 给定一个 $2^{n} \times 2^{n} \times 3$ 的彩色图像模式 $C I P$ 以及其灰度级别参数 $m$, 将编码结果存入队列集合 $Q=\left\{Q_{0}, Q_{1}, \ldots\right.$, $\left.Q_{3 m-1}\right\}$ 中*/

Step 1. 对于一个给定的大小为 $2^{n} \times 2^{n} \times 3$ 的彩色图像模式 CIP, 分别取得它的 $r, g, b$ 颜色分量组成的大小为 $2^{n} \times 2^{n}$ 的灰度图像模式 $M P[1], M P[2]$ 和 $M P$ [3], 并将用于计算矩形子模式的计数变量rect_num赋值为 0 .

Step 2. 用灰度图像的BPD方法依次将 3 幅灰度图像模式 $M P[1], M P[2]$ 和 $M P[3]$ 各自分解为 $m$ 幅二值图像 $B P_{i}(0 \leq i \leq m-1), B P_{i}(m \leq i \leq 2 m-1)$ 和 $B P_{i}(2 m \leq i \leq 3 m-1)$, 并将这 $3 m$ 幅二值图像 $B P_{i}(0 \leq i \leq 3 m-1)$ 连续存储; 最后将位面号 $i$ 赋值为 0 .

Step 3. 从二值图像模式 $B P_{i}$ 的第 1 个入口开始, 首先确定一个未被标识的矩形子模式的起始点 $(x, y)$, 再根据 矩形子模式的匹配(逆布局)算法来追迹相应的矩形子模式.

Step 4. 根据矩形子模式的效率尺度(即矩形子模式的面积)确定一个面积最大的矩形子模式,并将这个最 大的矩形子模式在二值图像模式 $B P_{i}$ 中作标识, 以便下一个起始点的寻找.

Step 5. 将矩形子模式的计数变量rect_num的值加 1, 并记录此最大矩形子模式的 3 个参数, 即起始点坐标 $(x, y)$ 、长度值 $L$ 和宽度值 $W$; 然后对起始点坐标 $(x, y)$ 作 $K$ 码降维变换,即 $S P \leftarrow K(x, y)$; 最后将 $S P, L$ 和 $W$ 这 3 个变量存储到 队列 $Q_{i}$ 中, 即有 $Q_{i}\{$ rect_num $\} \leftarrow\{S P, L, W\}$.

Step 6. 循环执行 Step 3 Step 5, 直到没有新的起始点为止.

Step 7. $i=i+1$. If $i \leq 3 m-1$, then go to Step 3, else go to Step 8 .

Step 8. 输出编码结果 $Q=\left\{Q_{0}, Q_{1}, \ldots, Q_{3 m-1}\right\}$.

解码算法的具体步骤如下:

$/ *$ 给定一个编码结果队列集合 $Q=\left\{Q_{0}, Q_{1}, \ldots, Q_{3 m-1}\right\}$ 、图像的灰度级别参数 $m$ 以及分辨率 $n$, 将解码结果存

入彩色图像模式 CIP 中,本算法中不妨以矩阵形式存放图像模式 $* 1$

Step 1. 将 3 幅灰度图像模式 $M P[1], M P[2]$ 和 $M P$ [3] (它们分别代表彩色图像的 $r, g, b$ 颜色分量)和 $3 m$ 幅二值图 像模式 $B P_{i}(0 \leq i \leq 3 m-1)$ 分别赋值为 $2^{n} \times 2^{n}$ 大小的全 $0($ 黑色)和全 1 (白色)的矩阵, 且将位面号 $i$ 武值为 0,3 幅灰度图像 模式组成的数组 $M P[j]$ 的下标变量 $j$ 赋值为 1 .

Step 2. 根据给定的编码队列集合 $Q$, 取得第 $i+(j-1) \times m$ 个位平面二值图像的编码结果, 即 $Q_{i+(j-1) \times m}$.

Step 3. 根据 $Q_{i+(j-1) \times m}$, 计算出该位平面二值图像的矩形子模式的总数rect_num, 对于其中的每个矩形, 依次获 取矩形的起始点坐标 $S P$ 、长度值 $L$ 和宽度值 $W$, 即有 $\{S P, L, W\} \leftarrow Q_{i+(j-1) \times m}\left\{r e c t \_n u m\right\}$; 然后对起始点坐标 $S P$ 作 $K$ 码升 维变换,即 $(x, y) \leftarrow K^{-1}(S P)$; 最后根据矩形子模式的 3 个参数 $(x, y) 、 L$ 和 $W$ 即可解码出该矩形子模式, 并将矩阵 $B P_{i+(j-1)}$ $\times_{m}$ 对应元素赋值为 0 (即黑色).

Step 4. $i=i+1$. If $i \leq m-1$, then go to Step 2, else go to Step 5 .

Step 5. 根据 $M P[j]=\sum_{i=0}^{m-1} 2^{i} B P_{i+(j-1) \times m}$, 即可解码出原始多值图像模式 $M P[j]$, 并将位面号 $i$ 赋值为 0 .

Step 6. $j=j+1$. If $j \leq 3$, then go to Step 2, else go to Step 7 .

Step 7. 根据 3 幅代表 $r, g$ 和 $b$ 颜色分量的灰度图像模式 $M P[1], M P[2]$ 和 $M P$ [3]即可解码出彩色图像模式 CIP.

\section{3 基于NAM的存储结构}

本节描述矩形子模式 $p=\{$ rectangle $\mid$ rectangle $=L \times W\}$ 情况下的NAM存储结构.因为一幅彩色图像模式可以分 解为 3 幅灰度图像模式 $M P[1], M P$ [2]和 $M P$ [3], 而每一幅灰度图像模式的输出是 $m$ 个顺序存储的队列 $Q=\left\{Q_{0}, Q_{1}, \ldots, Q_{m-1}\right\}$, 分别对应于 $m$ 幅位平面二值图像模式的 NAM表示, $Q_{i}(0 \leq i \leq m-1)$ 的构成主要由两个元素组 成, 即子模式的起始点 $S P$ 以及子模式的参数序列. 由于只记录 “black”像素, 因此起始点坐标不能省略, SP即为一 个坐标对 $(x, y), x$ 和 $y$ 的二进制码长度都为 $n$. 具体存储记录用 $K$ 码表示. $K$ 用相对值来记录, 本次记录的 $K$ 域用本 
次 $K$ 码减去上一个 $K$ 码的差值来记录, 即 $\Delta K=K_{i}-K_{i-1}$. 在统计意义下, 其长度为 $n$, 在实际情况下, 如果 $\Delta K$ 的长 度确实超过了 $n$, 则可以将该块拆分为两个块, 用两个记录来表示. 子模式的表示只有两个值, 即长和宽, 分别用 $L$ 和 $W$ 表示.按照 $K$ 的定义, $L$ 和 $W$ 的最大长度为 $n / 2$ 位.

综上所述, 彩色图像模式的编码结果的队列集合为 $Q=\left\{Q_{0}, Q_{1}, \ldots, Q_{3 m-1}\right\}$, 其中, $Q_{i}(0 \leq i \leq 3 m-1)$ 表可以分离存 储,也可以连续存储.存储结构如图 3 所示.

$$
\begin{array}{l|l|l|}
\hline Q_{i} \quad K & \{L, W\} \\
\hline
\end{array}
$$

Fig.3 The storage structure of NAM-based

图 3 基于 NAM 的存储结构

\section{4 算法的数据量分析}

对于一幅给定的大小为 $2^{n} \times 2^{n} \times 3$ 的彩色图像模式 CIP, 我们可以将其分解为 3 幅大小为 $2^{n} \times 2^{n}$ 的灰度图像模 式或者 $3 m$ 幅大小为 $2^{n} \times 2^{n}$ 的位平面二值图像模式 ( $m$ 是彩色图像的灰度级参数). 为了方便分析彩色图像模式表 示的数据量, 不妨令 $G$ 和 $F$ 分别表示任意的一幅灰度图像模式和一幅二值图像模式, 且其大小均为 $2^{n} \times 2^{n}$, 则 $G$ 和 $F$ 的像素总数 $N_{f}$ 和 $N_{f b}$ 也均为 $4^{n}$, 即 $N_{f}=4^{n}, N_{f b}=4^{n}$.

由于算法中被逆布局的子模式对象是任意大小的矩形 $p=\{$ rectangle $\mid$ rectangle $=L \times W\}$, 根据第 3.3 节的分析可 知,对于基于NAM的彩色图像表示方法来说,存储一个记录需占 $2 n$ 位,则其 $3 m$ 个位面的总数据量 $H_{b p d}$ 为

$$
H_{b p d}=\sum_{i=1}^{3} \sum_{j=1}^{m} H_{r}(i, j)=\sum_{i=1}^{3} \sum_{j=1}^{m} 2 n N_{r}(i, j)=\sum_{i=1}^{3} \sum_{j=1}^{m} 2 n N_{f b} C F(i, j) \frac{N_{r}(i, j)}{N_{L Q T B}(i, j)}=2 n 4^{n} \sum_{i=1}^{3} \sum_{j=1}^{m} C F(i, j) \frac{N_{r}(i, j)}{N_{\text {LQTB }}(i, j)}
$$

其中, $H_{r}(i, j), N_{r}(i, j), C F(i, j)$ 和 $N_{L Q T B}(i, j)$ 分别表示逆布局后第 $i$ 个色彩分量第 $j$ 个位面的总数据量、子模式总数、二 值图像的复杂度和用线性四元树表示的black节点总数.

对于基于线性四元树的彩色图像表示方法来说, 存储一个节点占 $3 n-1+m$ 位,则线性四元树的总数据量 $H_{L Q T}$ 为

$$
H_{L Q T}=\sum_{i=1}^{3}(3 n-1+m) N_{L Q T}(i)=\sum_{i=1}^{3}(3 n-1+m) N_{f} C p(i)=\sum_{i=1}^{3}(3 n-1+m) 4^{n} C p(i)=(3 n-1+m) 4^{n} \sum_{i=1}^{3} C p(i)
$$

其中, $N_{L Q T}(i)$ 表示第 $i$ 个色彩分量用线性四元树表示的总节点数, $C p(i)$ 表示第 $i$ 个色彩分量的图像复杂度.

假定 $\eta_{r b p d}, \eta_{L Q T}$ 分别为矩形NAM和线性四元树的压缩比,则有

$$
\begin{gathered}
\eta_{b p d}=\frac{3 m N_{f b}}{H_{b p d}}=\frac{3 m 4^{n}}{2 n 4^{n} \sum_{i=1}^{3} \sum_{j=1}^{m} C F(i, j) \frac{N_{r}(i, j)}{N_{\text {LQTB }}(i, j)}}=\frac{3 m}{2 n \sum_{i=1}^{3} \sum_{j=1}^{m} C F(i, j) \frac{N_{r}(i, j)}{N_{\text {LQTB }}(i, j)}} \\
\eta_{\text {LQT }}=\frac{3 m N_{f}}{H_{\text {LQT }}}=\frac{3 m 4^{n}}{(3 n-1+m) 4^{n} \sum_{i=1}^{3} C p(i)}=\frac{3 m}{(3 n-1+m) \sum_{i=1}^{3} C p(i)}
\end{gathered}
$$

设 $\varphi_{b p d L Q T}$ 为矩形NAM的压缩比与线性四元树的压缩比的比值,则有

$$
\varphi_{\text {bpdLQT }}=\frac{\eta_{b p d}}{\eta_{L Q T}}=\frac{(3 n-1+m) \sum_{i=1}^{3} C p(i)}{2 n \sum_{i=1}^{3} \sum_{j=1}^{m} C F(i, j) \frac{N_{r}(i, j)}{N_{L Q T B}(i, j)}}=\frac{(3 n-1+m) \sum_{i=1}^{3} \frac{N_{L Q T}(i)}{4^{n}}}{2 n \sum_{i=1}^{3} \sum_{j=1}^{m} \frac{N_{r}(i, j)}{4^{n}}}=\frac{(3 n-1+m) \sum_{i=1}^{3} N_{L Q T}(i)}{2 n \sum_{i=1}^{3} \sum_{j=1}^{m} N_{r}(i, j)}
$$

不难看出,第 1 组公式,即式(5)和式(6), 分别是矩形 NAM 和线性四元树的总数据量,它们都与图像模式的复 杂度有关. 图像模式的复杂度是参照线性四元树的块数来定义的,其目的主要是为了与线性四元树进行比较. 这 一组公式反映的是各自的绝对数据量,由于不同图像其复杂度是不同的,因此,其压缩效果也是不一样的.

第 2 组公式,即式(7)和式(8), 分别是矩形NAM和线性四元树各自的压缩比. 压缩比的定义为 $\eta=S_{m} / S_{n}$. 其 中, $S_{m}$ 为原始图像模式存储所占的总位数, $S_{n}$ 为具体算法处理后的图像模式所占的总位数.

最后一个公式,即式(9), 是矩形 NAM 的压缩比与线性四元树的压缩比的比值.此公式反映了矩形 NAM 和 
线性四元树之间的数据量的比值.通过这一比值, 可以比较矩形 NAM 相对于线性四元树的优劣.由于线性四元 树的分割是对称形的分割, 其分割方式受到很大的限制, 而 NAM 的分割是非对称的逆布局, 分割是最大限度 地形成子模式矩形,分割的灵活性更大. 由定理 3 可知, $\sum_{j=1}^{m} N_{r}(i, j)<N_{L Q T}(i)$, 从而可推出 $\sum_{i=1}^{3} \sum_{j=1}^{m} N_{r}(i, j)<$ $\sum_{i=1}^{3} N_{L Q T}(i)$. 因此, $\varphi_{b p d L Q T}>(3 n-1+m) / 2 n$. 也就是说,矩形 $N A M$ 的压缩比至少是线性四元树压缩比的 $(3 n-1+m) / 2 n$ 倍以上.比如在下一节的实验中, 当 $n=9, m=8$ 时, 从理论上来说, $\varphi_{b p d L Q T}>1.89$.

\section{5 实验结果及分析}

为了更好地说明基于NAM的彩色图像表示方法的有效性,本节从实验的角度来加以说明.实验中用来测试 的彩色图像模式的分辨率参数 $n=9$, 即大小为 $2^{9} \times 2^{9} \times 3$ 彩色图像模式,灰度级参数 $m=8$, 即 $2^{8}=256$ 级.

我们选择并给出了 4 幅彩色图像模式的实验结果,同时与流行的基于线性四元树的表示方法的实验结果 进行了比较.这 4 幅图像分别是 WaterLily,Gloriette,Flight 和 Lena, 如图 4 所示.这些图像具有较好的代表性, 能够 说明本方法的适应性. 相应的实验数据见表 1. 其中,Cc 为彩色图像的复杂度;NAM 为矩形 NAM 表示; Quadtree 为线性四元树表示, Ratio 为 NAM 与 Quadtree 的压缩因子之比.
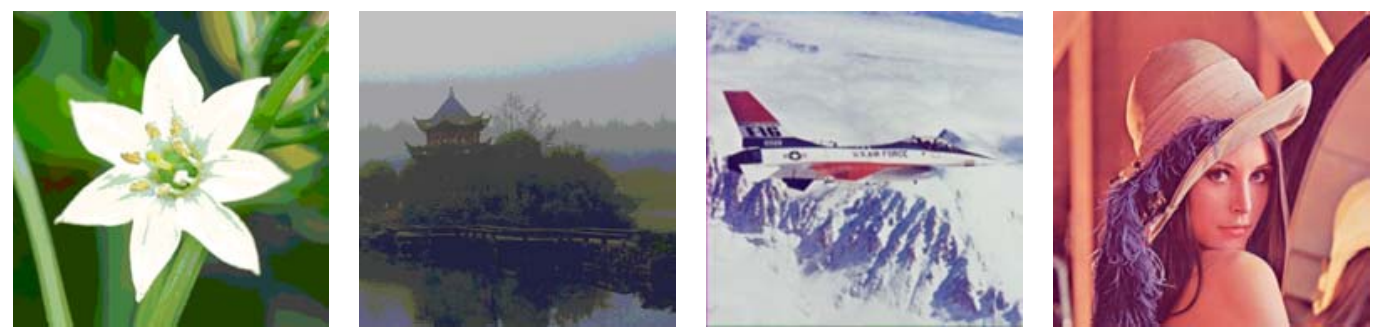

Fig.4 The original images of WaterLily, Gloriette, Flight, and Lena

图 4 WaterLily,Gloriette,Flight 和 Lena 的原始图像

Table 1 Comparison of the experimental data between NAM and Quadtree

表 1 NAM 和 Quadtree 的实验数据比较

\begin{tabular}{c|c|cc|ccc}
\hline \multirow{2}{*}{ Image } & \multirow{2}{*}{ Cc } & \multicolumn{2}{|c|}{ Number of subpatterns or nodes } & \multicolumn{3}{|c}{ Compression factor $\eta$} \\
\cline { 3 - 7 } & & NAM & Quadtree & NAM & Quadtree & Ratio \\
\hline WaterLily & 0.1953 & 57078 & 153621 & 6.12 & 1.20 & 5.10 \\
Gloriette & 0.2216 & 61140 & 174309 & 5.72 & 1.06 & 5.40 \\
Flight & 0.9119 & 589685 & 717153 & 0.59 & 0.26 & 2.27 \\
Lena & 0.9862 & 757957 & 775593 & 0.46 & 0.24 & 1.92 \\
\hline
\end{tabular}

从表 1 中 $C c$ 的数字来看,WaterLily 和 Gloriette 的复杂度相对较低, 而 Flight 和 Lena 的复杂度相对较高, 并且 4 幅图像的 Quadtree 表示的节点个数的多少也基本证实了这一点. 从本表中给出的 NAM 和 Quadtree 的子 模式数(节点数)的数据来看, NAM 方法在数据量方面的效果是非常明显的, 其子模式数远小于 Quadtree 方法的 节点数,对图像的适应性也非常好.

另外, 从表中压缩因子 $\eta$ 的数据来看, 当图像的复杂度较低时, 比如对 WaterLily和Gloriette来说,NAM和 Quadtree都具有数据压缩的能力, NAM的压缩比为 5.72 6.12, 而Quadtree的压缩比仅为 1.06 1.20, 显然, NAM的 压缩能力是Quadtree的 5.10 5.40 倍. 也即 $\varphi_{b p d L Q T}>1.89$. 显然, 这个实验结果证实了第 4 节的理论分析结果. 而当图 像的复杂度较高时, 比如对Flight和Lena来说, NAM和Quadtree都不具有数据压缩的能力, NAM的压缩比为 $0.46 \sim 0.59$, 而Quadtree的压缩比为 $0.24 \sim 0.26$, 但此时NAM的数据表示能力仍然是Quadtree的 1.92 2.27 倍. 也即 $\varphi_{b p d L Q T}>1.89$. 显然,这个实验结果也证实了第 4 节的理论分析结果.

综上所述,实验数据表明了理论分析的正确性.即无论图像的复杂程度如何,基于 NAM 的表示方法均优于 
基于线性四元树的表示方法,对图像具有较强的适应性.

因此,与流行的基于线性四元树的彩色图像表示方法相比,本文提出的基于 NAM 的表示方法能够更有效 地减少数据存储空间,是彩色图像模式表示的一种好方法.

\section{6 结论及展望}

借助于 Packing 问题的思想提出了一种基于 NAM 的彩色图像表示方法.该方法是一种全新的彩色图像模 式表示方法,旨在开拓一个全新的关于彩色图像模式表示的研究领域,其理论价值在于它的可扩展性,其实际应 用前景体现在它的数据压缩和表示上的可操作能力.理论分析和实验结果均表明,与流行的基于分层结构的四元 树彩色图像表示方法相比,本文的基于 NAM 的表示方法具有明显的优势,是彩色图像模式表示的一种好方法.

然而,尽管本文针对彩色图像模式做了较为深入的研究,但这些结果还是建立在单类型子模式(矩形)基础 之上的, 虽然即使是在这种情况下也能充分证明本方法的效能,但对于多子模式类型(如矩形与三角形等的组 合)来说, 我们预期将产生更少的子模式数并具有更强的数据表示及操作能力. 因此,多子模式类型的组合逆布 局是需要进一步研究的内容,其结果将会使基于 NAM 的彩色图像表示方法更具生命力.

\section{References:}

[1] Cline D, Egbert PK. Terrain decimation through quadtree morphing. IEEE Trans. on Visualization and Computer Graphics, 2001, 7(1):62-69.

[2] Gan XC, Wu SH, Tan Z. Image restoration based on spatial orientation tree. Journal of Computer Research and Development, 2001, 38(3):341-343 (in Chinese with English abstract).

[3] Jiao HL, Chen G. A color image fractal compression coding method. Journal of Software, 2003,14(4):864-868 (in Chinese with English abstract). http://www.jos.org.cn/1000-9825/14/864.htm

[4] Chen SK. An exact closed-form formula for $d$-dimensional quadtree decomposition of arbitrary hyperrectangles. IEEE Trans. on Knowledge and Data Engineering, 2006,18(6):784-798.

[5] Flusser J. Refined moment calculation using image block representation. IEEE Trans. on Image Processing, 2000,9(11):1977-1978.

[6] Liu ZY, Sun ZQ, Xu L, Peng X. Contour representation based on wedgelet. Journal of Systems Engineering and Electronics, 2006, 17(2):251-257.

[7] Du PJ, Fang T, Tang H, Shi PF. Encoding methods of spectral vector in hyperspectral remote sensing image. Journal of Shanghai University (English edition), 2005,9(1):52-57.

[8] Samet H. Hierarchical representations of collections of small rectangles. ACM Computing Surveys, 1988,20(4):271-309.

[9] Monasse P, Guichard F. Fast computation of a contrast-invariant image representation. IEEE Trans. on Image Processing, 2000,9(5): 860-872.

[10] Samet H. Data structures for quadtree approximation and compression. Communications of the ACM, 1985,28(9):973-993.

[11] Samet H, Webber RE. Storing a collection of polygons using quadtrees. ACM Trans. on Graphics, 1985,4(3):182-222.

[12] Li SX, Loew MH. The quadcode and its arithmetic. Communications of the ACM, 1987,30(7):621-626.

[13] Gargantini I. An effective way to represent quadtrees. Communications of the ACM, 1982,25(12):905-910.

[14] Szeliski R, Shum HY. Motion estimation with quadtree splines. IEEE Trans. on Pattern Analysis and Machine Intelligence, 1996, 18(12):1199-1210.

[15] Lee M, Samet H. Navigating through triangle meshes implemented as linear quadtrees. ACM Trans. on Graphics, 2000,19(2): 79-121.

[16] Rhee I, Martin GR, Muthukrishnan S, Packwood RA. Quadtree-Structured variable-size block-matching motion estimation with minimal error. IEEE Trans. on Circuits and Systems for Video Technology, 2000,10(1):42-50.

[17] He Z, Lee BS, Snapp R. Self-Tuning cost modeling of user-defined functions in an object-relational DBMS. ACM Trans. on Database Systems, 2005,30(3):812-853.

[18] Ji JF, Li S, Liu XH, Wu EH. View-Dependent progressive transmission of highly detailed surfaces. Journal of Software, 2006, 17(10):2192-2198 (in Chinese with English abstract). http://www.jos.org.cn/1000-9825/17/2192.htm 


\section{附中文参考文献:}

[2] 甘祥超,武栓虎,谈正.基于小波四叉树的图像去噪方法.计算机研究与发展,2001,38(3):341-343.

[3] 焦华龙,陈刚.一种分形彩色图像压缩编码方法.软件学报,2003,14(4):864-868. http://www.jos.org.cn/1000-9825/14/864.htm

[18] 冀俊峰,李胜,刘学慧, 吴恩华.细节高度复杂表面模型的视点相关渐进传输.软件学报,2006,17(10):2192-2198. http://www.jos. org.cn/1000-9825/17/2192.htm

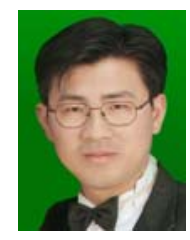

郑运平(1979-), 男, 湖北武汉人,博士生, 主要研究领域为计算机图形图像处理, 模 式识别。

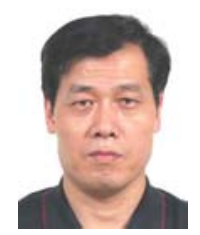

陈传波(1957-), 男, 博士, 教授, 博士生导 师, 主要研究领域为计算机网络, 信息工 程,图像处理,模式识别.

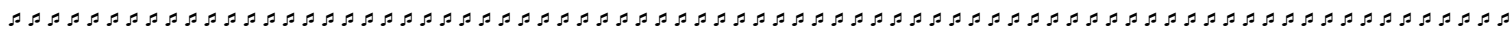

\section{Seventeenth International}

\section{World Wide Web Conference (WWW2008)}

\section{April 21-25, 2008}

The International World Wide Web Conferences Steering Committee (IW3C2) cordially invites you to participate in the 17th International World Wide Web Conference (WWW2008), to be held on April 21-25, 2008 in Beijing, China. The conference series has become the premier venue for academics and industry to present, demonstrate, and discuss the latest ideas about the Web. The technical program for the five-day conference will include refereed paper presentations, plenary sessions, panels, and poster sessions. The WWW2008 program will also include Tutorials and Workshops, a W3C track, a Developers track, a WWW in China track, and Exhibitions.

\section{IMPORTANT DATES}

\section{Submission Deadlines:}

Workshop Proposals: October 1, 2007

Refereed Papers: $\quad$ November 1, 2007 (HARD deadline; no extensions will be granted)

Tutorial Proposals: $\quad$ November 1, 2007

Posters: $\quad$ January 25, 2008 (estimated)

Acceptance Notification: Refereed Papers - January 15, 2008 (tentative)

Conference dates: $\quad$ April $21-25,2008$

\section{REFEREED PAPERS}

WWW2008 seeks original papers describing research in all areas of the Web. Topics include but are not limited to: Browsers and User Interfaces; Data Mining; Industrial Practice and Experience; Internet Monetization; Mobility; Performance and Scalability; Rich Media Search; Search Security and Privacy; Semantic Web; Social Networks and Web 2.0; Technology for Developing Regions; Web Engineering; XML and Web Data.

General queries regarding WWW2008 submissions can be sent to submissions@www2008.org. Other inquiries about the conference can be sent to info@www2008.orq.

The full call for papers, including detailed information about the scope of each track, formatting and submission requirements will be available soon from http://www2008.org. 